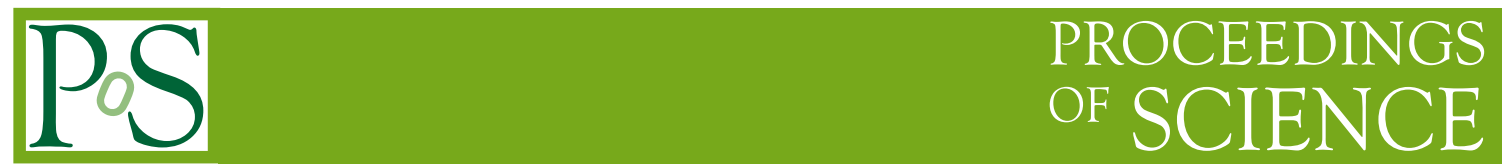

\title{
Rare B meson decays involving leptons at BABAR
}

\section{Giovanni ONORATO* (On behalf of the BABAR collaboration)}

INFN Napoli - Università "Federico II" Napoli

E-mail: giovannionorato@na.infn.it

We present recent results from BABAR on rare B meson decays involving leptons: searches of $B \rightarrow l \bar{v}_{l}, B \rightarrow K^{(*)} v \bar{v}$ and a model independent search of $B \rightarrow l \bar{v}_{l} \gamma$.

European Physical Society Europhysics Conference on High Energy Physics, EPS-HEP 2009, July 16 - 222009

Krakow, Poland

${ }^{*}$ Speaker. 


\section{Rare B meson decays involving leptons: theoretical framework}

\subsection{Leptonic B decays}

In the Standard Model (SM), the purely leptonic decay $B^{+} \rightarrow l^{+} v_{l}$ proceeds via quark annihilation into a $W^{+}$boson. The branching fraction is:

$$
\mathscr{B}\left(B^{+} \rightarrow l^{+} v_{l}\right)=\frac{G_{F}^{2} m_{B} m_{l}^{2}}{8 \pi}\left[1-\frac{m_{l}^{2}}{m_{B}^{2}}\right]^{2} \tau_{B}^{+} f_{B}^{2}\left|V_{u b}\right|^{2}
$$

In the contest of the SM, purely leptonic decays of the $B^{+}$meson are the only clean experimental methods of measuring $f_{B}$, assuming $\left|V_{u b}\right|$ from semileptonic $B \rightarrow u l v$ decays. Moreover they are sensitive to physics beyond the SM due to the possible insertions of New Physics (NP) heavy states in the annihilation process[1][2]. The radiative leptonic decay $B \rightarrow l v_{l} \gamma$ proceeds via quark annihilation into a virtual $W^{+}$boson after radiating a photon. The presence of the photon removes the helicity suppression, but introduces an additional suppression by a factor of $\alpha_{e m}$. The differential branching fraction versus photon energy $E_{\gamma}$ involves two form factors, $f_{V}$ and $f_{A}$ :

$$
\frac{d \mathscr{B}}{d E_{\gamma}}=\frac{\alpha_{E M} G_{F}^{2}\left|V_{u b}\right|^{2}}{48 \pi^{2}} m_{B}^{4}\left[f_{A}^{2}\left(E_{\gamma}\right)+f_{V}^{2}\left(E_{\gamma}\right)\right]\left(1-2 E_{\gamma} / m_{B}\right)\left(2 E_{\gamma} / m_{B}\right)^{3} .
$$

While the HQET model suggests $f_{A}=f_{V}$ [3], some models suggest $f_{A}=0[4]$.

\subsection{The $B \rightarrow K^{(*)} v \bar{v}$ decay}

The $b \rightarrow s v \bar{v}$ process proceeds via one-loop box or electroweak penguin diagrams. The SM predictions give branching fractions of $\mathscr{B}\left(B \rightarrow K^{*} v \bar{v}\right)_{S M}=\left(6.8_{-1.1}^{+1.0}\right) \times 10^{-6}$ and $\mathscr{B}(B \rightarrow$ $K v \bar{v})_{S M}=(4.5 \pm 0.7) \times 10^{-6}[6]$. However, many NP scenarios can enhance those values, where several mechanisms contribute to the rate: for example non-standard $Z^{0}$ coupling contributions could give an enhancement of up to a factor 10[5]. The kinematics of the decay is described in terms of $s_{v v}=m_{v v}^{2} / m_{B}^{2}$, where $m_{v v}$ is the invariant mass of the neutrino pair. NP effects can strongly affect the shape of the $s_{v v}$ distribution and this is taken into account to obtain a model independent limit.

\section{Experimental techinques and results}

\subsection{Untagged $B \rightarrow e / \mu v_{e / \mu}[7]$}

The $B \rightarrow l v_{l}$ has a strong signature given by the mono-energetic charged lepton in the rest B frame, with a momentum $p^{*} \approx m_{B} / 2$. We select the lepton candidate requiring $2.4 \mathrm{GeV} / \mathrm{c}<$ $p_{c . m} .<3.2 \mathrm{GeV} / \mathrm{c}$ and applying loose PID criteria. We combine all the charged particle and the neutral energy deposits in the calorimeter to reconstruct the other B. We use an extended maximum likelihood (ML) to extract signal and background yields, using simultaneously the Fisher output $p_{F I T}$ based on 5 variables and the energy-substituted mass $m_{E S}$, defined as $\sqrt{E_{\text {beam }}^{2}-\left|\vec{p}_{B}\right|^{2}}$. The procedure gives $18 \pm 18$ events with an efficiency of $(4.7 \pm 0.2) \%$ for the electron channel, and $1.4 \pm 17.2$ events with an efficiency of $(6.1 \pm 0.2) \%$ for the muon channel. We see no evidence of signal and we set an upper limit at $90 \%$ C.L.:

$$
\mathscr{B}\left(B^{+} \rightarrow \mu^{+} v_{m u}\right)<1.0 \times 10^{-6} \quad \mathscr{B}\left(B^{+} \rightarrow e^{+} v_{e}\right)<1.9 \times 10^{-6} .
$$




\section{$2.2 B \rightarrow l v_{l}$ with semileptonic $\operatorname{tag}[8]$}

The tagging technique consists in the reconstruction through semileptonic decays of one of the two B produced at the $\Upsilon(4 S)$. Once a B is reconstructed $\left(B_{T A G}\right)$, we search for the signal candidates in the recoil B $\left(B_{S I G}\right)$.

\subsection{1 $B \rightarrow \tau v$}

The $\tau$ particle is reconstructed through its main decays using PID and $\pi^{0}$ reconstruction: $\tau \rightarrow e \bar{v}_{e} v_{\tau}, \quad \tau \rightarrow \mu \bar{v}_{\mu} v_{\tau}, \quad \tau \rightarrow \pi v_{\tau}, \quad \tau \rightarrow \rho v_{\tau} \quad \rho \rightarrow \pi^{ \pm} \pi^{0}$. We use topological and kinematics variables to suppress background, and we fit the $E_{\text {extra }}$ distribution (defined as the sum of all unassigned cluster energy) to extract signal yields. The total number of expected background events is $521 \pm 31$, and the observed events are 610 , with a total efficiency of $\varepsilon=(10.54 \pm 0.41) \times 10^{-4}$. We obtain $\mathscr{B}\left(B \rightarrow \tau v_{\tau}\right)=(1.8 \pm 0.8 \pm 0.1) \times 10^{-4}$. Combining this result with the hadronic tagged analysis[9] $\left(\mathscr{B}\left(B \rightarrow \tau v_{\tau}\right)=(1.8 \pm 0.9 \pm 0.4) \times 10^{-4}\right)$ we have $\mathscr{B}\left(B \rightarrow \tau v_{\tau}\right)=(1.8 \pm 0.6) \times 10^{-4}$.

\subsection{2 $B \rightarrow e / \mu v_{e / \mu}$}

We select signal requiring $p^{*}>2.52(2.45)$ for electrons (muons), and fit the $E_{\text {extra }}$ shape. We see no evidence of signal in both channels, with $24 \pm 11$ expected events $(\varepsilon=(36.9 \pm 1.5) \times$ $\left.10^{-4}\right)$ and 17 observed events for electron, and $15 \pm 10$ expected $\left(\varepsilon=(27.1 \pm 1.2) \times 10^{-4}\right)$ with 11 observed for the muon channel. We set an upper limit at $90 \%$ C.L.:

$$
\mathscr{B}\left(B^{+} \rightarrow \mu^{+} v_{\mu}\right)<11 \times 10^{-6} \quad \mathscr{B}\left(B^{+} \rightarrow e^{+} v_{e}\right)<7.7 \times 10^{-6} .
$$

\section{$2.3 B \rightarrow l v_{l} \gamma$ with hadronic tag[10]}

We reconstruct the tag $\mathrm{B}$ in its most abundand charmed hadronic decays. We use a likelihood ratio based on 5 observables to reduce combinatorial background and search for one opposite charged lepton passing PID requirements; the $\gamma$ signal candidate is the most energetic neutral cluster; the missing momentum must be in the detector acceptance and we apply a $\pi^{+}, \omega$ and $\eta$ veto. We expect $2.7 \pm 0.4 \pm 0.4$ background events with an efficiency of $\varepsilon=(7.8 \pm 0.1 \pm 0.3) \times 10^{-4}$ and observe 4 events for the electron channel. We expect $3.4 \pm 0.7 \pm 0.7$ background events with an efficiency of $\varepsilon=(8.1 \pm 0.1 \pm 0.3) \times 10^{-4}$ and observe 7 events for the muon channel. The upper limit is

$$
\mathscr{B}\left(B^{+} \rightarrow \mu^{+} v_{m u} \gamma\right)<26 \times 26^{-6} \quad \mathscr{B}\left(B^{+} \rightarrow e^{+} v_{e} \gamma\right)<17 \times 10^{-6} \quad \mathscr{B}\left(B^{+} \rightarrow l^{+} v_{l} \gamma\right)<15 \times 10^{-6}
$$

We select in a more stringent but model dependent way the signal, requiring a cut on the angle between the lepton and the photon, and on the angle between the photon and the neutrino. We assume both the $f_{A}=f_{V}$ and the $f_{A}=0$ hypothesis. This reduce the efficiency in both modes and models by $40 \%$. The results are summarized in the following table

\begin{tabular}{||l||c|c|c||c|c|c||}
\hline \multicolumn{1}{|c||}{} & \multicolumn{3}{c||}{$f_{A}=f_{V}$ hypotesis } & \multicolumn{3}{c||}{$f_{A}=0$ hypotesis } \\
\hline & Expected & Observed & U.L. 90\% CL & Expected & Observed & U.L. 90\% CL \\
\hline$B \rightarrow e v \gamma$ & $0.6 \pm 0.1$ & 0 & $<8.4 \times 10^{-6}$ & $1.2 \pm 0.4$ & 3 & $<29 \times 10^{-6}$ \\
\hline$B \rightarrow \mu v \gamma$ & $1.0 \pm 0.4$ & 0 & $<6.7 \times 10^{-6}$ & $1.5 \pm 0.5$ & 2 & $<22 \times 10^{-6}$ \\
\hline Combined & & & $<3.0 \times 10^{-6}$ & & & $<18 \times 10^{-6}$ \\
\hline
\end{tabular}




\section{$2.4 B \rightarrow K^{(*)} v \bar{v}[11]$}

We reconstruct the tag $\mathrm{B}$ in both semileptonic and hadronic decay modes. We do not exploit the $\mathrm{K}$ kinematics in order to be model independent. We reconstruct the $K^{*+}$ through $K^{*+} \rightarrow K^{+} \pi^{0}$ decays and $K^{*+} \rightarrow K_{s}^{0} \pi^{+}$decays, and the $K^{0}$ through the $K^{0} \rightarrow K^{+} \pi^{-}$decay. We apply a loose selection based on topological observables, and we fit the $E_{\text {extra }}$ distribution to extract signal yields in the semileptonic tagged analysis. In the hadronic tagged analysis we fit the distribution of a Neural Network output based on topological variables. The results are summarized in the following table:

\begin{tabular}{|c|c|c|c|}
\hline U.L. 90\% C.L. & Hadronic & Semileptonic & Combined \\
\hline$B^{+} \rightarrow K^{*+} v \bar{v}$ & $21 \times 10^{-5}$ & $9 \times 10^{-5}$ & $8 \times 10^{-5}$ \\
\hline$B^{0} \rightarrow K^{0} v \bar{v}$ & $11 \times 10^{-5}$ & $18 \times 10^{-5}$ & $12 \times 10^{-5}$ \\
\hline
\end{tabular}

\section{Conclusions}

The searches for B decays involving leptons test the SM predictions and may be an indirect search for the New Physics in a way that is complementary for the direct search of New Physics at the high energy machines. No significant NP signal has been seen so far, but the experimental measurements can already set some constraint on parameters defining NP models.

\section{References}

[1] W. Hou Enhanced charged Higgs boson effects in $B^{-} \rightarrow \tau v^{-}, \quad \mu v^{-}$and $b \rightarrow \tau v^{-}+X$, Phys. Rev. D. 48, 2342-2344 (1993).

[2] A. G. Akeroyd and S. Recksiegel The effect of $H^{ \pm}$on $B^{ \pm} \rightarrow \tau^{ \pm} v_{\tau}$ and $B^{ \pm} \rightarrow \mu^{ \pm} v_{\mu}$, J. Phys. G: Nucl. Part. Phys. 292311 (2003).

[3] E. Lunghi, D. Pirjol and D. Wyler, Complex flavour couplings in supersimmetry and unexpected CP violation in the decay $B \rightarrow \phi K$, Nucl. Phys. B 649, 337349 (2003).

[4] G. Burdman, T. Goldman, and D. Wyler, Radiative leptonic decays of heavy mesons, Phys. Rev. D 51, 111 - 117 (1995).

[5] G Buchalla, G. Hiller and G. Isidori, Phenomenology of nonstandard Z couplings in exclusive semileptonic $b \rightarrow$ s transitions, Phys. Rev. D 63, 014015 (2000).

[6] W. Altmannshofer, A.J. Buras, D.M. Straub and M. Wick, New strategies for New Physics search in $B \rightarrow K^{*} v \bar{v}, \quad B \rightarrow K v \bar{v}$ and $B \rightarrow X_{s} v \bar{v}$ decays [hep-ph/arXiv:0902.0160v2].

[7] B. Aubert et al., Search for the rare leptonic decays $B^{+} \rightarrow l^{+} v_{l}(l=e, \mu)$, Phys. Rev. D 79, 091101(R) (2009).

[8] B. Aubert et al., A Search for $B^{+} \rightarrow \ell^{+} v_{\ell}$ Recoiling Against $B^{-} \rightarrow D^{0} \ell^{-} \bar{v} X$ hep-ex/arXiv:0809.4027.

[9] B. Aubert et al., Search for $B^{+} \rightarrow \tau^{+} v$ decays with hadronic B tags, Phys. Rev. D 77, 011107(R) (2008).

[10] B Aubert et al., A model-independent search for the decay $B \rightarrow l v \gamma$ hep-ex/0907.1681

[11] B Aubert et al., Search for $B \rightarrow K^{*} v \bar{v}$ decays, Phys. Rev. D 78, 072007 (2008). 